\title{
Relato de experiência das enfermeiras do Banco de Leite Humano da Santa Casa de Misericórdia de São Paulo, voltado às práticas de promoção, proteção e apoio ao aleitamento materno
}

\author{
Experience report from the nurses of the Human Milk Bank of Santa Casa de Misericórdia de \\ São Paulo, focused on practices of promotion, protection and support to breastfeeding
}

\author{
Claudia Tavares de Melo ${ }^{\circledR 1}$, Marta Bellazzi Padrão ${ }^{\circledR 1}$
}

\begin{abstract}
Resumo
Objetivo: Relatar a experiência das enfermeiras do banco de leite humano (BLH) da Santa Casa de Misericórdia de São Paulo, demonstrar as novas atribuições, descrever as repercussões destas mudanças e avaliar o volume de leite coletado. Método: Estudo transversal, descritivo e analítico. Os dados foram coletados dos arquivos de registros do BLH desta instituição, de 2016 a 2018. Resultado: Após início de um projeto com atividades educativas voltadas à divulgação da atuação do BLH, observamos o aumento do número de doadoras de leite materno e, consequentemente, melhoria no fornecimento de leite humano pasteurizado aos prematuros internados. No ano de 2016 os atendimentos somaram 2821 mães, e em 2018, 3238 (demonstrando um aumento de 15\% no período). No tocante ao volume de leite coletado, foram 278,65 litros em 2016, contra 370,91 litros em 2018; demonstrando um aumento de 33\%. Conclusão: As ações de acolhimento, marketing, socialização e busca ativa realizadas favoreceram o aumento do número de doadoras de leite materno e no volume de leite coletado, bem como sua fidelização. A ampliação das atividades levou a um importante espaço de atuação do enfermeiro que trabalha no BLH.
\end{abstract}

Palavras-chave: Aleitamento materno, Banco de leite, Enfermagem obstétrica

\footnotetext{
Abstract

Objective: To report an experience lived by the nurses of the human milk bank (HMB) of Santa Casa de Misericórdia de

1. Irmandade Santa Casa de Misericórdia de São Paulo. Banco de Leite Humano. São Paulo - SP - Brasil

Trabalho realizado: Irmandade Santa Casa de Misericórdia de São Paulo. Banco de Leite Humano. São Paulo - SP - Brasil Endereço para correspondência: Marta Bellazzi Padrão. Av. Águas de São Pedro, No 297 apto 82 -02302-070 - São Paulo SP - Brasil
}

São Paulo, demonstrate the new attributions, describe the repercussions of these changes and evaluate the amount of appointments and milk volume collected from 2016 to 2018. Method: It was a cross-sectional descriptive and analytical study. Results: After beginning a project with educational activities aimed disseminating the $H M B$, culminating in the increase in the number of breast milk donors and, consequently, in the milk provision (more regular) for hospitalized premature newborns. The total number of attendances jumped from 2821 in 2016 to 3238 in 2018 (an increase of 15\%). Regarding the volume of collected milk, we got 278,65 liters in 2016 against 370,91 liters in 2018 (an increase of $33 \%$ ). Conclusion: The actions of reception, marketing, socialization and the active search performed favored an increase in the number of breast milk donors and in the volume of collected milk, and to keep their loyalty. The expansion of these activities led to an important new field of nurses activities on the $H M B$.

Key words: Breast feeding, Milk banks, obstetric nursing

\section{Introdução}

No período de colonização do Brasil, era comum as mulheres delegarem a amamentação às amas de leite. Num primeiro momento às índias e, posteriormente, às negras escravas.

No início do século XX surgiu como alternativa às amas de leite, o emprego de fórmula e de leites modificados, fator que culminou em uma queda no aleitamento materno ${ }^{(1)}$.

Na década de 40 , foi montada a primeira estrutura operacional de um Banco de Leite Humano (BLH) no Brasil. Nesta época a regra era o desmame em prol da utilização dos produtos industrializados e a amamentação ocorria como exceção. Os BLH funcionavam como leiterias humanas, sendo um órgão de proteção social ${ }^{(1)}$.Num processo disruptivo, quando a política 
nacional buscava reverter o alto nível do desmame precoce, na década de 80, o Instituto Fernandes Figueira - Fiocruz, desenvolveu um "novo modelo" operacional de $\mathrm{BLH}$, rompendo o paradigma criado em 1943, transformando o BLH em unidade a serviço da amamentação e de execução de atividades de coleta, processamento e controle de qualidade ${ }^{(1)}$.

Hoje em dia a Fiocruz e a Rede Brasileira de Bancos de Leite promovem campanhas constantemente, em prol do aleitamento materno, incluindo ações assistenciais voltadas ao prematuro e ao recém-nascido baixo peso ao nascer.

A Irmandade Santa Casa de Misericórdia de São Paulo (ISCMSP), localizada na região central da cidade, prestando atendimento de diversas especialidades em seu complexo, é um hospital universitário de alta complexidade e de referência para gestantes de alto risco, possui um Banco de Leite Humano e trabalha de forma habilitada pela Rede Brasileira de Banco de Leite Humano. No ano 2000, destinou uma sala para a retirada de leite das lactantes com filhos internados na UTI (Unidade de Terapia Intensiva) neonatal (sala de ordenha) do mesmo hospital. Nesta época, houve uma demanda alta, pois, muitas mães de outros setores como UTI pediátrica e da Pediatria também queriam fazer uso da sala, tendo em vista extrair o leite para ajudar na recuperação do próprio filho. Com isso, e para evitar um fluxo que pudesse acarretar danos aos pacientes da UTI neonatal, foi estipulado que somente as mães da UTI neonatal poderiam usufruir da sala e outro local foi criado para tal. Contudo a Santa Casa começou o planejamento para a abertura de um BLH, visando atender a todas as crianças e mães que estivessem de passagem pelo hospital, a fim de coletar para ofertar leite materno para seus bebês prematuros na UTI Neonatal e de outros recém-nascidos internados na UTI Pediátrica.

O BLH foi inaugurado em 2014, com a ajuda do Rotary Foundation, com profissionais treinados, atualizados e com formação específica para desenvolver as rotinas necessárias.

Em 2016, iniciou-se o processo de pasteurização do leite coletado, estendendo-se o atendimento às doadoras moradoras das redondezas. Outro fato de destaque diz respeito à descoberta de que as funcionárias do hospital retornavam da licença de maternidade sem orientação sobre aleitamento materno. Isso fez com que se buscassem alternativas, tais como, a extração de leite em locais inadequados e o desmame precoce por meio medicamentoso.

Neste mesmo ano, iniciou-se o acolhimento dessas funcionárias, auxiliando no aleitamento à distância, com a extração de leite e acondicionamento de forma segura pelo BLH, para posterior transporte e oferta em casa, por conta da sua ausência laborativa.
Apesar de a Santa Casa possuir uma unidade nova de BLH, equipada e com profissionais qualificados para a atuação nesta área, a quantidade de leite coletado era insuficiente para atender a demanda dos pacientes internados na UTI Neonatal.

Afim de buscar a viabilização dos objetivos específicos, o BLH da Santa Casa passou a trabalhar além das nutrizes internadas no alojamento conjunto, com outras mães internadas no hospital, com as pacientes ambulatoriais e seus acompanhantes. Incluindo, funcionárias da instituição no período de amamentação ou doadoras voluntárias que comparecem no BLH.

Demais disso, o BLH é responsável por ações de promoção, proteção e apoio ao aleitamento materno exclusivo e execução de atividades de coleta da produção lática da nutriz, seleção, classificação, processamento, controle de qualidade e distribuição, sendo proibida a comercialização dos produtos por ele distribuídos ${ }^{(2-3)}$.

É responsabilidade do BLH as seguintes atividades $^{(2)}$ : desenvolvimento de ações de promoção, proteção e apoio ao aleitamento materno, como programas de incentivo e sensibilização sobre a doação de leite humano; prestação de assistência à gestante, puérpera, nutriz e lactente na prática do aleitamento materno; preparação da gestante para a amamentação; elaboração de medidas de prevenção de doenças e outros fatores que impeçam a amamentação ou a doação de leite humano ordenhado (LHO); orientações à puérpera e à nutriz sobre: os cuidados com a mama puerperal; cuidados ao amamentar; pega, posição e sucção; ordenha, coleta e armazenamento do Leite Humano Ordenado (LHO) em domicílio; cuidados na utilização do leite humano cru e pasteurizado (LHP); execução das operações de controle clínico da doadora; coleta, armazenagem e repasse do LHO para o BLH, se for Posto de Coleta de Leite Humano ( PCLH), ao qual está vinculado; registro das etapas e dos dados do processo, garantindo a rastreabilidade do produto; manutenção de um sistema de informação que assegure os registros relacionados às doadoras $\mathrm{e}$ os produtos, disponíveis às autoridades competentes, guardando sigilo e privacidade; estabelecimento de ações que permitam a rastreabilidade do LHO.

Tais atividades são executadas tanto pelo PCLH como BLH, porém o BLH também inclui processamento e distribuição do LHOP (Leite Humano Ordenhado e Pasteurizado) responde tecnicamente pelo processamento e controle de qualidade do LHO procedente do PCLH a ele vinculado; realiza o controle de qualidade dos produtos e processos sob sua responsabilidade ${ }^{(3)}$.

$\mathrm{O}$ trabalho do enfermeiro em banco de leite humano não reflete apenas em extrair ou orientar a extração do leite materno, ele é um profissional que tem múltiplas capacitações e habilidades intra e extra- 
-hospitalares ${ }^{(4)}$. Além disso, cabe destacar, a título de informação e em complemento à introdução exposta que a enfermagem segue regramento próprio, consubstanciado na Lei do Exercício Profissional (Lei ${ }^{\circ}$ 7.498/1986) e seu Decreto Regulamentador (Decreto $\mathrm{n}^{\circ}$ 94.406/1987), além do Código de Ética dos Profissionais de Enfermagem ${ }^{(4)}$.

Neste sentido, a enfermagem atua na promoção, prevenção, recuperação e reabilitação da saúde humana, com autonomia e em consonância com os preceitos éticos e legais. É responsabilidade do enfermeiro seguir as determinações do Centro de Referência Nacional por Bancos de Leite Humano - Instituto Fernandes Figueira, Fundação Oswaldo Cruz, Ministério da Saúde em suas Normas Técnicas para Bancos de Leite Humano.

Anualmente em todo o mundo, cerca de $30 \mathrm{mi}-$ lhões de bebês nascem prematuros ou com baixo peso e que adoecem logo nos primeiros dias de vida, Segundo o relatório de coalizão global, que inclui o Fundo das Nações Unidas para a Infância (UNICEF) e a Organização Mundial da Saúde (OMS) em torno de 2,5 milhões de recém-nascidos morreram nos primeiros 28 dias de vida, a maioria por causas evitáveis, dentre elas a ausência de leite materno ${ }^{(5)}$.

Segundo a OMS, em 2017 o Brasil teve 40 partos prematuros por hora, ou seja, 931 prematuros nascidos por dia, sendo o maior índice na região nordeste e a menor no Sudeste. Nosso país é o décimo entre os de maior índice de partos prematuros segundo o Ministério da Saúde ${ }^{(9)}$. Na ISCMSP no período de julho de 2016 a dezembro de 2018, nasceram 503 prematuros segundo as estatísticas internas do setor de internação da Santa Casa.

A ISCMSP atende mulheres moradoras em áreas livres, que não realizam pré-natal adequado, com antecedentes de uso de álcool e de substâncias ilícitas, múltiplos parceiros, presença de contexto sócio econômico e cultural de violência, em especial a mulher, tornando o papel da equipe multidisciplinar fundamental e desafiador, fortalecendo o autocuidado e a orientação em especial quando falamos do binômio mãe-filho. Este perfil também deve ser levado em consideração quando se fala em cuidados na área da saúde. Quando existe a desinformação, entra o trabalho do enfermeiro no processo de cuidar, de informar, fortalecendo o indivíduo.

Silva e Berto ${ }^{(6)}$ relatam que se deve estimular à gestante e/ou puérpera a falar de suas dificuldades, conversar sobre os mitos mais comuns; ajudá-la na construção do conhecimento (esclarecendo concepções confusas, explicar o processo de amamentação, mostrar vídeos, discutir vantagens e desvantagens sobre do aleitamento materno, conversar sobre amamentação com grupos de mães, dentre outros).
Há vários motivos para as mães doarem leite materno, Alencar, Seild ${ }^{(7)}$ citam em seu estudo os fatores que levaram as mães doarem o leite materno: está a expressão de doação como ajuda a outras mães impossibilitadas de amamentar; o excesso de produção láctea, superior à necessidade do bebê; experiência prévia de dificuldade e/ou impedimento de amamentação da própria doadora.

\section{Objetivos}

\section{Geral}

Relatar a experiência de enfermeiras da Santa Casa de Misericórdia de São Paulo, e caracterizar as ações por elas desenvolvidas no BLH.

\section{Específico}

Analisar ao longo de 2016 a 2018 o número de atendimentos e volume de leite coletado.

\section{Materiais e Método}

Estudo transversal, descritivo e analítico realizado no período de 2016 a 2018 no BLH da Santa Casa de Misericórdia de São Paulo, hospital universitário, localizado em São Paulo. Os dados foram coletados dos arquivos de registros do BLH do referido hospital.

A coleta de dados Ocorreu após a aprovação do Comitê de Ética da Santa Casa de Misericórdia de São Paulo - CAAE 30623619.70005479 versão 1 e da Comissão de ética da Santa Casa de Misericórdia de São Paulo n ${ }^{\circ} 5479$.

\section{Resultados}

Em função da falta de doadoras de leite, iniciou-se um projeto com várias atividades educativas e de conscientização da equipe multiprofissional no hospital (palestras, grupos de conversa, aulas, visitas ao BLH) voltado à divulgação das atividades desenvolvidas no BLH, ressaltando a importância do aleitamento e da doação de leite materno realizadas em nosso complexo hospitalar além das atividades técnicas realizadas pelos enfermeiros na captação do leite.

Em 2016 iniciou-se a distribuição de folders e divulgação do BLH no hospital, campanhas de incentivo ao Aleitamento Materno e foi realizada a VIII Semana de Amamentação voltada a educação em serviço aos profissionais de diversas categorias.

A distribuição de folders foi mantida em 2017 pelos diversos setores do hospital incluindo a Faculdade de Ciências Médicas da Santa Casa de São Paulo e a Escola de Enfermagem da Irmandade. Iniciamos, com 
anúncios nos meios de comunicação interna e distribuição de banners pelo hospital central.

Realizada a IX Semana de Amamentação no ano de 2017 voltada à educação continuada da equipe de enfermagem e nutrição, com distribuição de lembrancinhas para as doadoras (pacientes internadas, externas e funcionárias) de leite materno.

Foi iniciado, também, um processo de busca ativa nas unidades do hospital com ações realizadas no leito.

Em 2018 foram proferidas palestras nos cursos de Enfermagem e Medicina da faculdade da Santa Casa de Misericórdia São Paulo, e foram intensificadas as mensagens e informações no servidor e no mês de agosto deste ano, foi trabalhada a conscientização do aleitamento em toda a instituição.

A X Semana de Amamentação que ocorreu no ano de 2019 teve como foco o incentivo ao aleitamento com a participação ativa das mães com a troca de saberes e informações no Alojamento Conjunto e para outras mulheres internadas. Foram criados impressos digitais pequenos em outras línguas como o inglês, francês e espanhol para facilitar a comunicação com o grande número de pacientes estrangeiras atendidas em nosso hospital.

As palestras foram adaptadas a rodas de conversas no próprio setor em termos de práticas educativas com temas voltados a saúde, amamentação, doação de leite, apoio qualificado e humanizado frente ao cuidado do bebê e ao cuidado da mulher e mãe.

Foi realizada a abertura de campo para enfermeiras em aprimoramento em enfermagem saúde materno-infantil no BLH bem como visitas direcionadas de alunos e residentes da instituição. Neste mesmo ano foi firmada uma parceria com PCLH do Hospital HCOR.
Na Tabela 1 apresentamos a evolução no número de atendimentos realizados no BLH de 2016 a 2018. Destaca-se, que no ano de 2018 a Santa Casa, passou por uma crise muito grande e neste período o quadro de profissionais foi reduzido em $50 \%$ bem como o fornecimento de material e medicamentos, fato que acabou reduzindo a quantidade de pacientes no BLH.

Na tabela 2 observamos que o volume de leite coletado, aumentou de 13,27\% em 2017 em relação a 2016 e 28,15\% entre os anos de 2016 a 2018.

Na tabela 3 apresentamos o número de pacientes e a origem das mesmas dos diversos setores da instituição, destacando o Pronto Socorro (Central, Infantil, Ginecologia e Obstetrícia), Departamento de Medicina (DM) onde internamos os casos clínicos, Departamento de Ortopedia e Traumatologia (DOT), Departamento de Ginecologia e Obstetrícia (DOGI). Nas salas de pós-parto do Centro Obstétrico (CO), onde as mães ficam aguardando leitos de internação por problemas de superlotação nas enfermarias, Departamento de Cirurgia (DC) e Ambulatórios.

\section{Discussão}

O acolhimento, estratégias de divulgação e campanhas educativas foram os três pilares positivos em nossa experiência. Sobre o acolhimento, o bom atendimento das doadoras foi apontado como possibilidade de idealizá-las, além da ampliação de coletas, facilitando o retorno da doadora. A experiência e conhecimento dos enfermeiros foi um diferencial no atendimento, removendo barreiras, crenças e tabus que dificultam a doação.

\begin{tabular}{|c|c|c|c|c|c|c|}
\hline \multirow{3}{*}{$\begin{array}{l}\text { MÊS } \\
\text { Janeiro }\end{array}$} & \multicolumn{6}{|c|}{ ANO } \\
\hline & \multicolumn{2}{|c|}{2016} & \multicolumn{2}{|c|}{2017} & \multicolumn{2}{|c|}{2018} \\
\hline & 255 & $9,04 \%$ & 187 & $5,66 \%$ & 231 & $7,13 \%$ \\
\hline Fevereiro & 247 & $8,76 \%$ & 233 & $7,05 \%$ & 274 & $8,46 \%$ \\
\hline Março & 299 & $10,60 \%$ & 239 & $7,24 \%$ & 207 & $6,39 \%$ \\
\hline Abril & 267 & $9,46 \%$ & 168 & $5,09 \%$ & 189 & $5,84 \%$ \\
\hline Maio & 236 & $8,37 \%$ & 195 & $5,90 \%$ & 194 & $5,99 \%$ \\
\hline Junho & 216 & $7,66 \%$ & 311 & $9,42 \%$ & 221 & $6,83 \%$ \\
\hline Julho & 246 & $8,72 \%$ & 317 & $9,60 \%$ & 317 & $9,79 \%$ \\
\hline Agosto & 341 & $12,09 \%$ & 364 & $11,02 \%$ & 479 & $14,79 \%$ \\
\hline Setembro & 164 & $5,81 \%$ & 406 & $12,29 \%$ & 410 & $12,66 \%$ \\
\hline Outubro & 192 & $6,81 \%$ & 322 & $9,75 \%$ & 347 & $10,72 \%$ \\
\hline Novembro & 155 & $5,49 \%$ & 291 & $8,81 \%$ & 219 & $6,76 \%$ \\
\hline Dezembro & 203 & $7,20 \%$ & 270 & $8,17 \%$ & 150 & $4,63 \%$ \\
\hline Total & 2821 & $100,00 \%$ & 3303 & $100,00 \%$ & 3238 & $100,00 \%$ \\
\hline
\end{tabular}


Litros de leite coletados no Banco de Leite Humano na ISCMSP de 2016 a 2018 .Brasil, 2018

\begin{tabular}{|c|c|c|c|c|c|c|}
\hline \multirow{3}{*}{$\begin{array}{l}\text { MÊS } \\
\text { Janeiro }\end{array}$} & \multicolumn{6}{|c|}{ ANO } \\
\hline & \multicolumn{2}{|c|}{2016} & \multicolumn{2}{|c|}{2017} & \multicolumn{2}{|c|}{2018} \\
\hline & 20,96 & $7,52 \%$ & 19,4 & $5,55 \%$ & 22,1 & $5,96 \%$ \\
\hline Fevereiro & 20,16 & $7,23 \%$ & 21 & $6,01 \%$ & 26,01 & $7,01 \%$ \\
\hline Março & 22,97 & $8,24 \%$ & 23 & $6,58 \%$ & 19,5 & $5,26 \%$ \\
\hline Abril & 20,02 & $7,18 \%$ & 15,6 & $4,46 \%$ & 19,1 & $5,15 \%$ \\
\hline Maio & 20,53 & $7,37 \%$ & 17,83 & $5,10 \%$ & 29,7 & $8,01 \%$ \\
\hline Junho & 18,86 & $6,77 \%$ & 39,7 & $11,35 \%$ & 31,8 & $8,57 \%$ \\
\hline Julho & 26,34 & $9,45 \%$ & 36,2 & $10,35 \%$ & 38,7 & $10,43 \%$ \\
\hline Agosto & 33,87 & $12,16 \%$ & 43,12 & $12,33 \%$ & 55,6 & $14,99 \%$ \\
\hline Setembro & 20,59 & $7,39 \%$ & 37,98 & $10,86 \%$ & 44,3 & $11,94 \%$ \\
\hline Outubro & 16,06 & $5,76 \%$ & 27,1 & $7,75 \%$ & 40,9 & $11,03 \%$ \\
\hline Novembro & 34,19 & $12,27 \%$ & 34 & $9,72 \%$ & 29,4 & $7,93 \%$ \\
\hline Dezembro & 24,1 & $8,65 \%$ & 34,7 & $9,92 \%$ & 13,8 & $3,72 \%$ \\
\hline Total & 278,65 & $100,00 \%$ & 349,63 & $100,00 \%$ & 370,91 & $100,00 \%$ \\
\hline
\end{tabular}

Atendimento de pacientes para coleta de leite humano em setores diversos na ISCMSP, de 2017 a 2018 . Brasil, 2018.

\begin{tabular}{|c|c|c|c|c|c|c|}
\hline \multirow{2}{*}{ Local } & \multicolumn{2}{|c|}{2017} & \multicolumn{2}{|c|}{2018} & \multicolumn{2}{|c|}{ Total } \\
\hline & $N$ & $\%$ & $N$ & $\%$ & $N$ & $\%$ \\
\hline PSI & 1 & $6,25 \%$ & 0 & $0,00 \%$ & 1 & $3,13 \%$ \\
\hline PSC & 1 & $6,25 \%$ & 6 & $37,50 \%$ & 7 & $21,88 \%$ \\
\hline PSDOGI & 2 & $12,50 \%$ & 0 & $0,00 \%$ & 2 & $6,25 \%$ \\
\hline DM & 3 & $18,75 \%$ & 4 & $25,00 \%$ & 7 & $21,88 \%$ \\
\hline DOT & 4 & $25,00 \%$ & 2 & $12,50 \%$ & 6 & $18,75 \%$ \\
\hline DOGI & 1 & $6,25 \%$ & 1 & $6,25 \%$ & 2 & $6,25 \%$ \\
\hline $\mathrm{CO}$ & 0 & $0,00 \%$ & 2 & $12,50 \%$ & 2 & $6,25 \%$ \\
\hline DC & 4 & $25,00 \%$ & 0 & $0,00 \%$ & 4 & $12,50 \%$ \\
\hline Ambulatórios & 0 & $0,00 \%$ & 1 & $6,25 \%$ & 1 & $3,13 \%$ \\
\hline Total & 16 & $100,00 \%$ & 16 & $100,00 \%$ & 32 & $100,00 \%$ \\
\hline
\end{tabular}

As campanhas educativas direcionadas a população intra-hospitalar são estratégias pontuais que ocorrem por um determinado tempo e por alguma razão específica como informes no servidor ou cartazes no hospital, com bons resultados durante e logo após a sua execução, contudo essas medidas acabam sendo esquecidas ou perdem o impacto ou a valorização ao longo de um tempo.

A educação direcionada para categorias com conhecimentos científicos específicos, encoraja os futuros profissionais, incentivadores ou formadores de opinião enfocando a doação como um ato responsável de cunho social foram mais eficientes.

Campanhas motivadas pela força do altruísmo, em que se combinam o senso de recompensa pessoal e o desejo de ajudar os bebês prematuros, são bem aceitas entre as nutrizes e parecem que tem um poder de sensibilizar muito mais a população adulta.

Como demonstrado na tabela 2 há aumento no volume de leite coletado após o das campanhas educativas.

A pesquisa Nascer no Brasil, um inquérito nacional sobre parto e nascimento da Escola Nacional de Saúde Pública Ensp/Fiocruz ,mostrou o excesso de intervenções obstétricas e o baixo uso de boas práticas na atenção ao parto e revelaram que a taxa de prematuridade brasileira (11,5\%) é quase duas vezes superior à observada nos países europeus. Muitos 
casos podem decorrer de uma prematuridade iatrogênica, ou seja, retirados sem indicação, em mulheres com cesarianas agendadas ou avaliação incorreta da idade gestacional. O estudo alertou para possíveis consequências. "A prematuridade se constitui no maior fator de risco para o recém-nascido adoecer e morrer não apenas imediatamente após o nascimento, mas também durante a infância e na vida adulta. Os prejuízos extrapolam o campo da saúde física e atinge as dimensões cognitivas e comportamentais, tornando esse problema um dos maiores desafios para a Saúde Pública contemporânea.

No Brasil a taxa de cesariana é de 36\%, na Irmandade Santa Casa de Misericórdia de São Paulo (que é um hospital de referência em gestação de alto risco), a taxa é de $42,46 \%$ e de nascidos prematuros $14 \%$ segundo dados do Setor de Internação.

O impacto do aleitamento materno na primeira hora de vida, na sobrevivência neonatal e infantil foi estudo de vários trabalhos e comprova o papel fundamental na redução dos índices apontados.

As estatísticas mostram que as doações de leite não acompanham o aumento do número de partos prematuros no mesmo período. Muitos hospitais enfrentam dificuldades em suprir a demanda principalmente, porque os BLH tem uma atuação não lucrativa e é proibitiva a função de comercialização no Brasil. Portanto, o marketing é importante para a captação de mulheres doadoras de leite, sendo necessária a utilização de estratégias consistentes para tornar a doação de leite parte de hábitos e valores da população.

A doação de leite não faz parte da vida da maioria da população feminina por isso, é fundamental o planejamento, o desenvolvimento, a avaliação de estratégias e a sua socialização, possibilitando novas formas de captação.

É importante a criação de um ensino voltado a esta finalidade desde o início da vida escolar. O Brasil não se prepara para captar doadoras e nutrizes. Temos que construir as doadoras do futuro. Os jovens deveriam aprender nas escolas desde pequenos a importância da amamentação bem como a doação de leite materno. Com isso, ampliasse a visão sobre o tema, socializando este princípio, visando a contribuição de nutrizes no seu período de lactação desenvolvendo a responsabilidade social.

Os enfermeiros devem valorizar a relação com as mães, estimulando o resgate da prática; "as orientações devem começar a partir da realidade de cada uma das mães, determinando prioridades, e tendo em vista suas necessidades e recursos". O enfermeiro deve ajudá-la no momento do desespero e desistência da prática da amamentação, sendo esta atitude importante para a sua continuidade ${ }^{(8)}$

$\mathrm{O}$ acolhimento realizado pelos enfermeiros do
BLH nas buscas ativas com as pacientes internadas no alojamento conjunto e em outras unidades do hospital, resultou em aumento na adesão dessas a doação de leite materno e ao aleitamento à distância para as mães com incapacidade transitória de amamentar.

\section{Conclusão}

Concluímos que o volume de leite coletado, aumentou de 13,27\% em 2016/2017 para 28,15\% em 2018.

No ano de 2016 os atendimentos no banco de leite foram de 2821 mães, em 2018 somamos 3238 atendimentos e tivemos um aumento de $15 \%$ no período. No tocante ao volume de leite coletado, foram 278,65 litros em 2016, comparado a 370,91 litros em 2018; demonstrando um aumento de 33\% em 2018, em relação a 2016.

A ampliação das atividades levou a um importante espaço de atuação do enfermeiro que trabalha no $\mathrm{BLH}$, culminando no aumento do número de doadoras de leite materno, no volume de leite coletado e fornecimento mais regular de leite aos prematuros, ampliando o benefício aos pacientes bem como no aumento da quantidade de leite disponibilizado aos que necessitam da sua utilização com os seus benefícios intrínsecos.

A ação de acolhimento, marketing, socialização do banco e das buscas ativas realizadas pelo hospital fizeram a diferença, para a captação de doadoras e de sua fidelização.

O bom atendimento a doadora é uma estratégia eficaz e necessita da capacitação e da boa vontade dos profissionais. A oferta de melhores condições, horários mais flexíveis e disponibilidade de coletas externas, também devem fazem parte do atendimento do banco de leite.

\section{Referências}

1. Almeida JAG, Novak FR Amamentação: um híbrido naturezacultura J Pediatr (Rio J.). 2004; 80(5 suppl):s119-s125.

2. Hinrichsen SL. Banco de leite humano. In: Hinrichsen SL. Biossegurança e controle de infecções: risco sanitário hospitalar. Rio de Janeiro: Medsi; 2004. p.153-7.

3. Rocha APM, Castro CRA, Souza MX, Messias CM. Enfermeiro e a mulher no ciclo gravídico-puerperal: prática educativa entre amamentação X Banco de Leite. Rev Pesq Cuid Fundam Online. [Internet]. 2010. (Ed. Supl.):978-82. [citado 2019 Jun 30]. Disponível em: http://www.seer.unirio.br/index.php/ cuidadofundamental/article/view/1230/pdf_345

4. Conselho Federal de Enfermagem. Resolução COFEN n 311/2017. [Internet]. Aprova o código de ética dos profissionais de enfermagem [citado 2019 Abr 12]. Disponivel: www. portalcofen.gov.br/2019

5. Nações Unidas. OMS: cerca de 30 milhões de bebês nascem prematuros por ano no mundo. [Internet]. [citado 2019 Nov 15]. Disponível em: https://nacoesunidas.org/oms-cerca-de30-milhoes-de-bebes-nascem-prematuros-por-ano-no-mundo/ 
6. Silva ML, Berto NRT. Avaliação das condições de ordenha domiciliar em um banco de leite humano em Cascavel PR. Monografia (Trabalho de Conclusão de Curso). [Internet]. Cascavel: Fundação Assis Gurgacz; 2008. [2019 Out 15]. Disponível em: http://www.fag.edu.br/tcc/2008/ Nutri\%E7\%E3o/avaliacao_das_condicoes_de_ordenha_ domiciliar_em_um_banco_de_leite.pdf.

7. Alencar LCE, Seild EMF. Percepção de mulheres doadoras sobre a prática de doação de leite humano: promoção e incentivo para o banco de leite. [Internet]. [citado 2018 Out 14]. Disponível em: http://www.furb.br/formularios/aleitamento/anais/blhs/ art_blhs_01.pdf.
8. Frota MA, Aderaldo NNS, Silveira VG, Rolim KMC, Marins MC.O reflexo da orientação na prática do aleitamento materno. Cogitare Enferm. 2008; 13(3):403-9.

9. Brasil. Ministério da Saúde. O Brasil está entre os dez países com o maior número de partos prematuros, aponta OMS. 2012. [Internet]. [2019 Set 20]. Disponível em: http:/ / www.brasil.gov. br/noticias/arquivos/2012/05/04/brasil-estaentre-os-dezpaises-com-o-maior-numero-departos-prematuros-aponta-oms

Trabalho recebido: $16 / 12 / 2019$

Trabalho aprovado: $22 / 09 / 2020$

Trabalho publicado: 23/09/2020 